Vol. 16, $n^{\circ} 1 \mid 2012$

Varia

\title{
Maia Clarissa Nunes, Neto Flávio de Sá, Costa Marcos, Bretas Marcos Luiz, (coords.). História das prisões do Brasil
}

2 vols., Rio de Janeiro, Editora Rocco, 2009. $1^{\circ}$ vol. : 316 p. ; $2^{\circ}$ vol. : 319 p., ISBN 9788532524478.

\section{Tiago Pires Marques}

\section{(2) OpenEdition}

\section{Journals}

Édition électronique

URL : https://journals.openedition.org/chs/1344

DOI : $10.4000 /$ chs. 1344

ISSN : 1663-4837

\section{Éditeur}

Librairie Droz

\section{Édition imprimée}

Date de publication : 1 mai 2012

Pagination : 136-141

ISBN : 978-2-600-01594-3

ISSN : 1422-0857

\section{Référence électronique}

Tiago Pires Marques, «Maia Clarissa Nunes, Neto Flávio de Sá, Costa Marcos, Bretas Marcos Luiz, (coords.). História das prisões do Brasil », Crime, Histoire \& Sociétés / Crime, History \& Societies [En ligne], Vol. 16, n¹ | 2012, mis en ligne le 12 mars 2013, consulté le 23 mars 2022. URL : http:// journals.openedition.org/chs/1344; DOI : https://doi.org/10.4000/chs.1344

Ce document a été généré automatiquement le 23 mars 2022

(C) Droz 


\section{Maia Clarissa Nunes, Neto Flávio de Sá, Costa Marcos, Bretas Marcos Luiz, (coords.). História das prisões do Brasil}

2 vols., Rio de Janeiro, Editora Rocco, 2009. $1^{\circ}$ vol. : 316 p. ; $2^{\circ}$ vol. : 319 p., ISBN 9788532524478.

Tiago Pires Marques

\section{RÉFÉRENCE}

Maia Clarissa Nunes, Neto Flávio de Sá, Costa Marcos, Bretas Marcos Luiz, (coords.).

História das prisões do Brasil, 2 vols., Rio de Janeiro, Editora Rocco, 2009. $1^{\circ}$ vol. : 316 p. ; $2^{\text {o }}$ vol. : 319 p., ISBN 9788532524478.

1 L'histoire des prisons au Brésil, du XVIII ${ }^{\mathrm{e}}$ siècle à nos jours, constitue le prétexte et le thème de fond de cet ouvrage collectif, qui rassemble dix-sept chapitres, divisés en deux volumes. Plusieurs générations de chercheurs croisent leurs travaux dans cette somme, certains d'entre eux montrent les premiers résultats d'une enquête, d'autres s'appuient sur d'importants parcours de recherches dans les domaines de l'histoire et de la sociologie des normativités et des institutions pénales. Si, en règle générale, il n'est pas chose aisée de recenser un ouvrage collectif, dans ce cas, l'ambition et la qualité inégale des articles la rend particulièrement ingrate. En effet, cet inventaire ne rendra pas justice à tous les articles en laissant dans l'ombre quelques éléments susceptibles de critique, mais aussi des textes qui mériteraient d'être mis en avant.

2 Considérons d'abord les grandes lignes de force de cette Histoire des prisons au Brésil. L'introduction, signée par le collectif des coordinateurs, nous permet de comprendre les objectifs de l'ouvrage, ses contenus, et nous instruit sur l'état de la recherche dans ce domaine au Brésil. Les auteurs rappellent que ce pays compte de grands 
établissements pénitentiaires, caractérisés par une crise chronique et marqués par une histoire troublée que les médias retranscrivent au quotidien. Cette visibilité sociale et politique de la question pénale apparait comme singulière au Brésil, au moins dans ce degré d'importance et en comparaison avec les cas européens. C'est sans doute pour cette raison que le problème des représentations médiatiques et sociales de l'univers des prisons fait l'objet d'une attention récurrente au fil de ces articles. Et, pourtant, à la présence médiatique et sociale de la prison dans la société brésilienne ne correspond pas, dans l'espace universitaire et de la recherche, une attention proportionnelle. Malgré certains travaux de qualité, pointés comme des références dans ce domaine (e.g. Fernando Salla ${ }^{1}$; Regina Célia Pedroso ${ }^{2}$; Elizabeth Cancelli $\left.{ }^{3}\right)$, il n'existait pas, nous informent les auteurs, d'ouvrages historiques comparant les institutions dans les différentes régions. Ce livre se propose de combler cette lacune.

Les auteurs passent en revue différentes méthodologies d'analyse mobilisables pour aborder ce champ d'études, des classiques Rusche et Kirchheimer, Gersham Sykes, Goffman et Foucault à des auteurs plus récents, tels que Pieter Spierenburg et David Garland. Réfléchissant sur l'impact considérable de l'historiographie marxiste et des travaux de Michel Foucault au Brésil, les auteurs prennent leurs distances par rapport à Foucault, jugé porteur de l'idée d'une prison utilisée avec efficacité comme instrument de contrôle social des classes populaires par les élites dirigeantes. L'application du récit foucaldien au cas du Brésil emporterait l'affirmation 1) que ces institutions transportent et appliquent une logique de discipline sociale, et 2) que ce programme est efficace. Or, comme de toute évidence le point 2) est inexact, les auteurs concluent par l'inapplicabilité du modèle foucaldien au Brésil. Toutefois, on peut se demander si cette lecture, qui fait écho aux critiques des interprétations foucaldiennes depuis les années 1980, ne repose pas, en réalité, sur une interprétation biaisée de Surveiller et punir. En effet, un élément central de l'analyse foucaldienne c'est justement l'idée que l'échec de la fonction de discipline est un élément décisif de la logique pénitentiaire. En réalité, pour Foucault, la prison construit les délinquants en tant que personnage social et groupe ciblé par les institutions de l'Etat. Leur incapacité à les "corriger» aboutit paradoxalement à légitimer et à justifier leur maintien. En outre, plusieurs articles de ces deux volumes semblent plutôt renforcer quelques points clefs des travaux de Foucault. Certes, il faut ici tenir compte d'une chronologie décalée et d'éléments propres à la réalité historique brésilienne. Mais n'est-ce pas toujours le cas lorsqu'il s'agit d'appliquer à un pays donné une clef de lecture historique forgée à partir de l'étude d'une autre société ? On peut signaler, à l'endroit de la logique disciplinaire au Brésil, le remplacement progressif de la ritualisation publique des punitions principalement la peine capitale, les châtiments corporels (appliqués aux esclaves) et le travail forcé - par la prison, celle-ci élevée au rang d'élément symbolique de la modernité dans l'espace public (vol. I, 259, 268). Dans la même ligne de pensée, on observe aussi la contemporanéité de la montée de la logique juridique et politique du libéralisme et de l'organisation de l'économie capitaliste (vol. I, 44), ainsi que l'émergence de l'Etat médico-légal en association étroite avec un projet scientifique d'objectivation et de naturalisation de la personnalité du délinquant (vol. I, 55).

4 En revanche, l'ensemble des articles présentés permet aussi de voir que si les projets de réforme pénitentiaire ont eu une fonction symbolique au sein d'un programme de modernisation comprenant le disciplinaire (au sens foucaldien), d'autres rationalités ont eu une place non moins importante dans le domaine de la justice pénale. Cet aspect devient frappant lorsque l'on tient compte de la diversité des institutions pénales qui 
ont coexisté au XIXe siècle. Il faut tenir compte, tout d'abord, des lieux de détention et d'application des peines hérités de l'époque coloniale : le Calabouço, un donjon destiné aux esclaves, et l'Aljube, un bâtiment ecclésiastique adapté à la détention temporaire de criminels présumés, pendant la première moitié du XIX ${ }^{\mathrm{e}}$ siècle (tous les deux situés à Rio de Janeiro) ; le bateau-prison (Presiganga) utilisé entre 1808 et 1831, un des remparts de la société esclavagiste; ou encore l'île-prison Fernando de Noronha, avec son organisation particulière, étrangère aux principes d'administration pénitentiaire importés des États-Unis et de l'Europe. Et il faut surtout mettre en avant la coexistence d'un système de justice privée, appliquée aux esclaves, et d'un système public fondé sur les principes du libéralisme, les deux parfois se recoupant, notamment dans l'application des châtiments aux esclaves par des entités publiques sous délégation des seigneurs (vol. I, 188 ; vol. I, 220). Peut-on donc se servir de Foucault pour analyser le cas brésilien? La réponse est forcément oui et non, comme on vient de le voir. En tout cas, la discussion sur l'applicabilité du modèle foucaldien ne me semble pas d'un grand intérêt dans l'élucidation empirique de l'histoire du système pénal au Brésil. La question Foucault pourrait éventuellement être considérée comme métahistorique, car dépendante de la valorisation relative des éléments inclus dans la définition du modèle disciplinaire.

5 Dans l'introduction de l'ouvrage, les auteurs soutiennent, contre les récits des progrès de la civilisation désuets depuis longtemps, mais aussi contre les focalisations excessives sur les débats autour de la réforme des prisons issues des approches marxistes et foucaldiennes, la nécessité d'une grille d'analyse mettant en avant d'autres éléments : le processus politique conduisant du modèle idéal à sa mise en œuvre; et la documentation des prisons permettant d'observer leur administration. Afin de systématiser les éléments d'une telle grille d'analyse, les auteurs s'appuient sur un texte de John A. Conley, qui prône l'analyse détaillée de trois moments ou phases dans le développement des établissements pénitentiaires : la conception du projet, le modèle idéal; la concrétisation pratique du modèle et ce que ce processus implique d'une redéfinition politique du modèle même; et sa fonctionnalité au quotidien (vol. I, 18-19) ${ }^{4}$. Bien que plusieurs articles soient en effet sensibles à ces trois dimensions, ce cadre fait davantage l'objet d'un énoncé qui est lui-même de l'ordre de l'idéal que d'une utilisation systématique dans la recherche ou dans l'organisation du livre.

6 Par son caractère introductif et général, le premier article, signé par Carlos Aguirre, mérite une attention particulière. Coordinateur (en collaboration) d'un ouvrage de référence sur les prisons en Amérique latine, Aguirre ${ }^{5}$ nous donne un aperçu intéressant de la place des prisons dans les systèmes pénaux au Brésil et au Mexique. En se concentrant sur la structure économique et de classe de ces sociétés, ainsi que sur les divisions socio-juridiques (entre individus libres et esclaves) et les divisions raciales, Aguirre intègre la prison dans une histoire politique et sociale qui soustrait d'emblée la question pénale du registre abstrait de l'histoire des institutions et des idées morales. Certains passages de son analyse contiennent des généralisations qui ajoutent peu à la compréhension historique que nous avons aujourd'hui du monde carcéral moderne, comme l'explication, quelque peu mécanique, du développement des pénitenciers par l'ordre capitaliste. Mais l'article a le mérite de montrer la coopération des différentes logiques de punition et discipline et leur articulation avec les formes, moderne et prémoderne, de maintien de l'ordre racial et d'organisation du travail. L'article montre également la part considérable de la prison dans les mécanismes de contrôle social des sociétés concernées, bien que partagée avec d'autres institutions, en particulier 
l'armée. Ainsi, nous apprenons, par exemple, que dans la seconde moitié du XIX siècle, le système pénitentiaire brésilien comptait, à son apogée, 10000 prisonniers, tandis que l'armée avait en charge entre 8000 et 12000 individus considérés comme des criminels, contraints d'intégrer ses rangs à titre punitif (49). L'article précise également les caractéristiques de la population carcérale : principalement des groupes autochtones, des Noirs et des métis, la vie dans la prison prolongeant les divisions sociales et raciales de la société libre. L'auteur conclut de façon convaincante que la prison renforçait les structures socio-raciales dominantes et servait l'ordre économique (61).

7 L'article d'Aguirre est donc une excellente porte d'entrée dans la question des systèmes de punition légale en Amérique latine, et notamment au Brésil. Un second article aborde le débat sur la réforme pénitentiaire, mettant en perspective l'interdépendance entre les idées juridiques et les sentiments religieux (vol. I, 79-108). Trois articles traitent des formes de punitions en marge du système carcéral moderne: les institutions mentionnées supra du bateau-prison (Presiganga) (vol. I, 109-134), l'îleprison Fernando de Noronha (vol. I, 135-178) et les châtiments corporels appliqués aux esclaves dans les prisons de São Paulo au XIX ${ }^{e}$ siècle (vol. I, 179-216). Un sixième article, l'un des plus intéressants de ces deux volumes, examine la relation entre le système de l'esclavage urbain et le système pénitentiaire à Rio de Janeiro (vol. I, 217-252). L'auteur examine l'utilisation de main-d'œuvre esclave dans la construction d'infrastructures pénitentiaires dans la capitale brésilienne. Cette tutelle publique d'esclaves remis temporairement à des institutions de l'Etat moyennant des compensations aux seigneurs est désignée, par son auteur, sous le terme de double captivité (vol. I, 220).

8 Les autres articles sont des études de cas portant sur diverses questions à propos des institutions pénales brésiliennes. Dans les deux volumes, cinq articles se concentrent sur des institutions à Rio de Janeiro, de la fin du XVIII ${ }^{e}$ siècle aux premières décennies $\mathrm{du} \mathrm{XX} \mathrm{X}^{\mathrm{e}}$ siècle. Ces articles analysent des espaces pénaux précis : la Maison de Correction à Rio de Janeiro, inaugurée en 1850 (vol. I, 217-252 ; vol. I, 283-314; vol. II, 7-46) ; le Calabouço (le donjon, en fonction jusqu'en 1878) et l'Aljube (1808-1856) (vol. I, 253-282). Dans le deuxième volume, un article décrit la mise en place du système pénitentiaire dans l'État de Rio Grande do Sul (vol. II, 47-74) ; trois articles sont consacrés aux prisons et établissements correctionnels de Recife, la capitale de l'État de Pernambuco (vol. II, 75-110; vol. II, 111-154; vol. II, 249-276); et un article collectif examine le développement du système pénitentiaire dans l'État de Ceará (vol. II, 155-184). Marcos Bretas analyse le système de représentations des prisons dans la littérature et la culture populaire aux XIXe et XXe siècles (vol. II, 185-214). La prison se pose en lieu séparé de la société, espace caché et mystérieux ; ce système se caractérise donc, selon l'auteur, par la tension entre ce caché et les investissements imaginaires, parfois soutenus par des témoignages directs, d'écrivains et journalistes. Cet article montre que ces récits, qui dépeignent des détenus tel des «sauvages » inadaptés à la vie sociale civilisée, tout en niant le potentiel réformateur des prisons, avaient un rôle important dans la légitimation du système pénitentiaire (vol II, 191). Dans un autre article, Peter M. Beattie examine le débat sur la sexualité et la politique des visites intimes en prison du début du XXe siècle à nos jours (vol. II, 215-248). Enfin, un dernier texte donne un aperçu de la présence au Brésil du positivisme criminologique à la fin du XIX siècle et, un peu plus tard, de la biotypologie et de certains propos eugéniques (vol. II, 277-317).

Abordant un enjeu important mais souvent négligé par les chercheurs, l'article de Peter $M$. Beattie mérite ici une attention particulière. Dans le travail de Lemos Britto, un 
important pénaliste brésilien du début du $\mathrm{XX}^{\mathrm{e}}$ siècle, Beattie trouve quelques-uns des principaux thèmes du discours des pénalistes sur le problème de la sexualité des détenus et de la sexualité en prison: la prise de conscience de ce que l'abstinence sexuelle imposée constitue une deuxième peine, ajoutée à la privation de la liberté ; l'idée que l'abstinence sexuelle peut être nocive à la santé mentale et qu'elle mène à l'homosexualité et à la masturbation, et se compte donc parmi les facteurs de "démoralisation». L'auteur montre comment ces notions s'articulaient avec les représentations sexuelles dominantes, à leur tour, dépendantes de considérations concernant la productivité, la discipline et la violence. Jusqu'aux années 1980, bien que faisant l'objet des débats des experts tout au long du XXe siècle, la question sexuelle a été évitée et chapeautée par l'idée que la sexualité des détenus devrait être domptée par l'éducation morale et par le travail. La situation a changé avec la loi de 1984, établissant le système de visites intimes pour les détenus (hommes et femmes). Actuellement dissociée de la préoccupation de correction morale, la question sexuelle est devenue, par le biais de ce système de visites, l'une des principales monnaies d'échange avec les détenus lorsqu'il s'agit d'aborder l'épineux problème de l'ordre et de la discipline dans des prisons surpeuplés aux conditions de vie très dégradées. En fait, la négociation de ces visites s'avère beaucoup moins onéreuse et plus efficace que l'imposition de mesures disciplinaires, la construction de nouvelles prisons ou la centième tentative de réforme des institutions (vol. II, 244).

En résumé, les deux volumes de cette Histoire des prisons au Brésil constituent un ouvrage qui mérite, sans aucun doute, d'être pris en compte. Pourtant, il manque d'une organisation claire et de systématicité. Une chronologie comparée des différents États à l'examen et un index thématique auraient contribué à intégrer les résultats. Il n'est donc pas question d'une histoire du système de justice pénale au Brésil, couvrant l'ensemble du territoire de façon équilibrée et traitant de façon exhaustive une série de questions prédéfinies, telle que le titre pourrait laisser espérer. Mais très peu de pays disposent, à présent, d'un tel panorama historique et ce livre, qui reflète probablement un paysage de recherche éclaté et déployé sur des campus éloignés, est, pour le cas brésilien, un premier pas très méritoire. Outre l'intérêt évident qu'il présente pour les chercheurs travaillant sur le Brésil, ce livre fournit également des éléments pertinents dans le cadre d'une sociologie historique des systèmes pénaux à une échelle élargie. Nous nous référons en particulier au problème de la coopération entre le système punitif et disciplinaire associé à l'esclavage et le système pénitentiaire moderne. Cette coopération s'est traduite par une multitude de statuts civils au XIX ${ }^{e}$ siècle - citoyen libre, esclave, esclave libéré, Africain libre sous tutelle, esclave temporairement sous contrôle de l'État (vol. I, 181, 188, 264, 266) - créant un système normatif et symbolique complexe, dont les effets de structure sur l'univers carcéral du Brésil mériteraient maintenant une analyse approfondie. Particulièrement utile dans l'attention accordée à l'intégration des structures punitives typiques des sociétés coloniales de l'Ancien Régime à des institutions juridiques et pénales créées dans le cadre de projets de modernité, ce recueil d'articles donne, par conséquent, d'importants indices pour comprendre un problème historico-sociologique qui dépasse le cas du Brésil. 


\section{NOTES}

1. Salla, Fernando, As prisões de São Paulo, São Paulo, Annablume, 1999.

2. Pedroso, Regina Célia, Os signos da opressão. História e violência nas prisões brasileiras, São Paulo, Arquivo do Estado/ Imprensa Oficial do estado, 2003.

3. Cancelli, Elizabeth, Carandiru. A prisão, o psiquiatra e o preso, Brasília, Editora da UnB, 2005.

4. Conley, John A., "L'histoire des prisons aux États-Unis : proposition pour une méthode de recherche”, in Petit, Jacques-Guy, La Prison, le Bagne et l'Histoire, Genève, Librairie des Méridiens, 1984.

5. Aguirre, Carlos, Salvatore, Ricardo D., (eds.), The Birth of the Penitentiary in Latin America. Essays on criminology, prison reform, and social control, 1830-1940, Austin, University of Texas Press, 1996.

\section{AUTEURS}

\section{TIAGO PIRES MARQUES}

CERMES3 - Groupe CESAMES (Université Paris-Descartes)

Centro de Estudos de História Religiosa (Universidade Católica Portuguesa)

tiago.marques@parisdescartes.fr 